\title{
Treatment outcomes and prognostic factors for non- malignancy associated secondary hemophagocytic lymphohistiocytosis in children
}

\section{Hua Pan}

Affliated hospital of qingdao university

\section{Gaoyan Wang}

Affiliated hospital of qingdao university

\section{Enben Guan}

Affiliated hospital of qingdao university

\section{Liang Song}

Affiliated hospital of qingdao university

\section{Aiqin Song}

Affiliated hospital of qingdao university

Xiaodan Liu

Affiliated hospital of qingdao university

Zhi Yi

Affiliated hospital of qingdao university

Li-rong Sun ( $\nabla$ sunlr@vip.sina.com )

\section{Research article}

Keywords: Hemophagocytic lymphohistiocytosis, prognostic factor, risk stratification

Posted Date: February 18th, 2020

DOl: https://doi.org/10.21203/rs.2.23890/v1

License: (c) (i) This work is licensed under a Creative Commons Attribution 4.0 International License.

Read Full License 


\section{Abstract}

Background: Secondary hemophagocytic lymphohistiocytosis $(H L H)$ is a rare hyperinflammatory syndrome that requires prompt diagnosis and appropriate treatment. A risk-stratification model that could be used to identify high-risk pediatric patients with HLH who should be considered for second-line therapies, including salvage regimens and allogeneic hematopoietic cell transplantation (HCT), was developed.

Methods: The medical records of 88 pediatric patients (median age 1.4 years, range 0.2-15 years) with non-malignancy associated secondary HLH were retrospectively reviewed. Treatment strategies included dexamethasone, etoposide, and cyclosporine.

Results:Survival analysis showed patients with Epstein-Barr virus(EBV)-HLH experienced better 5-year overall survival (OS) than patients with HLH due to other infections and unknown causes, autoimmune disease, or immunodeficiency ( $80 \%$ vs. $77 \%, 65 \%, 11 \%, p<0.001$ ). On multivariate analysis, among all patients, non-response at 8 weeks was the most powerful predictor of poor OS. When treatment response was excluded, hemoglobin $<60 \mathrm{~g} / \mathrm{L}$ and albumin $<25 \mathrm{~g} / \mathrm{L}$ at diagnosis were associated with poor OS. In patients with EBV-HLH, hemoglobin $<60 \mathrm{~g} / \mathrm{L}$ at diagnosis was associated with poor OS. A prognostic risk score was established and weighted based on hazard ratios calculated for three parameters measured at diagnosis: hemoglobin $<60 \mathrm{~g} / \mathrm{L}$ ( 2 points), platelets $<30 \times 10^{9} / \mathrm{L}$ ( 1 point), albumin $<25 \mathrm{~g} / \mathrm{L}$ ( 2 points). Fiveyear OS of low-risk (score $0-1$ ), intermediate-risk (score 2), and poor-risk (score $\geq 3$ ) patients were $88 \%$, $38 \%$, and $22 \%$, respectively $(p<0.001)$.

Conclusions: These findings indicate that clinicians should be aware of predictive factors at diagnosis and consider 8-week treatment response to identify patients with high-risk of disease progression and the need for second-line therapy and allogeneic HCT.

\section{Background}

Hemophagocytic lymphohistiocytosis $(\mathrm{HLH})$ is a potentially fatal condition of genetic or functional hypercytokinemia that is characterized by uncontrolled proliferation of activated lymphocytes and macrophages. HLH is associated with a wide spectrum of signs and symptoms, including fever, hepatosplenomegaly, pancytopenias, hypertriglyceridemia, hypofibrinogenemia, neurological symptoms and pathological findings of hemophagocytosis in the bone marrow or other organs[1]. HLH is a lifethreatening condition. Prompt diagnosis and initiation of treatment with dexamethasone and etoposide are essential[1]. Treatment of HLH can be associated with high morbidity and mortality, including severe sepsis and multi-organ failure in relapsed/refractory patients[1].

There are two major subtypes of HLH, which have been classified as "primary HLH" and "secondary HLH" based on the age of disease onset, family history, and the identification of a series of genetic variations that are responsible for the decreased functions of T/NK cells, such as PRF1/STXBP2/ITK[2]. Recently, Jordan et al proposed two concepts to clarify how HLH is diagnosed and treated: within the broader 
syndrome of HLH, "HLH disease" should be distinguished from "HLH disease mimics" and HLH subtypes should be categorized by specific etiologic associations, not an ambiguous dichotomy of "primary" and "secondary"[3].

Traditionally, secondary HLH is considered a rare hyperinflammatory syndrome that occurs in individuals without a family history or underlying genetic defect. Secondary HLH may be triggered by infections, rheumatologic diseases, malignancy, acquired immune deficiency states, and drugs[4]. Diagnosis and initiation of treatment for patients with secondary HLH is difficult because signs and symptoms overlap with several chronic conditions, including sepsis, multi-organ dysfunction, and progression of malignancies or rheumatic disease[5-6].

There remains an umet need to increase clinicians' knowledge of HLH. In particular, clinicians must be aware of factors that are predictive of treatment response and survival in patients with HLH. Currently, data describing relevant prognostic factors and risk-stratification in children with secondary HLH are scarce[7-8]. The objectives of this retrospective study were to evaluate treatment response and mortality and identify predictive factors for treatment response and survival in children with non-malignancy associated secondary HLH. A risk-stratification model that could be used to identify high-risk pediatric patients with HLH who should be considered for second-line therapies, including salvage regimens and allogeneic HCT, was developed.

\section{Methods}

\section{Study subjects}

Children diagnosed with HLH between 2005 and 2018 according to HLH-2004 criteria (fever, splenomegaly, bicytopenia, hypertriglyceridemia and/or hypofibrinogenemia, hemophagocytosis, ferritin $\geq 500 \mu \mathrm{g} / \mathrm{L}$, low/absent NK-cell activity, and soluble CD25 [s/L-2r] [ $\geq 2400 \mathrm{U} / \mathrm{ml}$ ], an affected sibling, and/or a molecular diagnosis based on familial hemophagocytic syndrome (FHL)-causative genes were eligible for his study[4]. The majority of patients satisfied at least $5 / 6$ of these criteria as NK-cell activity and soluble CD25 were not evaluated. Exclusion criteria were 1) diagnostic criteria not met or 2) malignancy-associated HLH (MA-HLH). This research was conducted in accordance with the guidelines of the Ethics Committee of the Affiliated Hospital of Qingdao University.

\section{Parameters associated with HLH}

The medical records of the included patients were retrospectively reviewed. Pretreatment complete blood cell (CBC) count, levels of fibrinogen and albumin, and prothrombin time (PT) were extracted from serial laboratory analyses.

Clinical outcomes were assessed based on the highest ferritin, aspartate aminotransferase (AST), alanine aminotransferase (ALT), total bilirubin, lactate dehydrogenase (LDH), and triglyceride levels recorded during the 4 weeks after treatment initiation. 
Epstein-Barr virus (EBV)-association was evaluated by viremia (including low level chronic viremia) and symptomatology of EBV infection, serologic tests (EBV early antigen [EA], EBV nuclear antigen [EBNA], EBV viral capsid antigen [VCA] IgG/lgM) and measuring EBV DNA level using real time polymerase chain reaction (RQ-PCR).

\section{Treatments}

Treatment strategies were based on the HLH-94 protocol, and included dexamethasone, etoposide, and cyclosporine $[5,6,14]$. If complete response was achieved at week 8 , patients were provided maintenance therapy, while patients with known familial disease or persistent nonfamilial disease proceeded to continuation therapy.

Treatment response at 4 weeks and 8 weeks after treatment, and dynamic changes during the 8 weeks of treatment were evaluated. Complete response was defined as resolution of all clinical signs and symptoms, CBC recovery, and normalization of abnormal laboratory findings associated with HLH. Partial response was defined as either $\mathrm{CBC}$ recovery or normalization of laboratory findings. Progressive disease was defined as persistence of cytopenia and abnormal laboratory findings.

Early stable responders were defined as patients with a complete response at both 4 and 8 weeks after treatment. Late stable responders were defined as patients who failed to achieve complete response at 4 weeks after treatment but showed a continuous response until 8 weeks after treatment. Unstable responders were defined as patients who showed a transient response and eventually progressed to 8 weeks after treatment. Non-responders were defined as patients exhibiting no response (See Supplemental methods for Statistical analysis).

\section{Results}

\section{Baseline characteristics}

The medical records of 88 patients (median age 1.4 years, range $0.2-15$ years) with non-malignancy associated secondary HLH were retrospectively reviewed. Causes of HLH were: EBV-associated $(n=30$ : recent primary infection $n=15$, chronic active EBV infection $n=3$, past infection $n=12$ ), unknown cause $(n=19)$, infection other than EBV $(n=20$ :cytomegalovirus $n=10$, brucella $n=3$, Staphylococcus aureus $n$ $=2$, Streptococcus pneumoniae $n=2$, Mycoplasm $n=1$, parvovirus $n=1$, herpes simplex $n=1$ ), autoimmune disease $(n=13$ : systemic lupus erythematosus $n=4$, juvenile rheumatoid arthritis $n=5$, dermatomyositis $n=1$, Kawasaki disease $n=2$, anaphylactoid purpura $n=1)$, or immunodeficiency $(n=6$ : chronic granuloma $n=2$, X-linked lymphocyte proliferative diseases $n=2$, humoral immune deficiency $n=$ 2). Baseline characteristics and treatment response for the included patients are summarized in Table 1. Fever was observed in all patients and 49 (55.7\%) patients had splenomegaly. The median day of the peak values of ferritin is 7 days before initiation. 
Table 1

Baseline characteristics and treatment outcomes of included patients $(n=88)$.

\section{Characteristic}

Gender (male), \%

Age, years old

Fever

$\mathrm{CMV} \%$

Leukocyte

Neutrophil

Platelet

Hemoglobin

Fibrinogen

Albumin

Ferritin

AST

ALT

LDH

TG

CRP

GGT

D-dimer

DIC \%

Splenomegaly \%

Jaundice \%

Bone marrow involvement \%

Hepatomegaly \%
Value

$50(56.8 \%)$

$1.4(0.2-15)$

$88(100 \%)$

$13(14.8 \%)$

$3.22(0.25-36.47)$

$1.195(0.05-11.86)$

$67.00(7-562)$

$86.5(31-131)$

$1.29(0.27-4.56)$

29.27

5.61

3564.5 (132.0-591123.0)

$249.15(21.0-9420.0)$

207.50 (8.0-4953.0)

944.5 (156.0-5311.0)

$3.34(0.39-13.56)$

$15.05(0.25-161.54)$

$207.5(7.0-1550.0)$

$2489.0(129.0-35600.0)$

$28(31.8 \%)$

$49(55.7 \%)$

$34(38.6 \%)$

$51(58.0 \%)$

$65(73.9 \%)$

Abbreviations: $\mathrm{HLH}$, hemophagocytic lymphohistiocytosis; AST, aspartate aminotransferase; ALT, alanine transferase; LDH, lactate dehydrogenase; CRP, C-reactive protein; DIC, disseminated intravascular coagulation; EBV, Epstein-Barr virus; CR, complete remission; EBV, Epstein-Barr virus; TG, triglyceride; GGT, gamma-glutamyl transpeptidase. 


\section{Characteristic}

Ascites \%

Lymphadenopathy \%

CR achievement after treatments

Therapy

HLH94/2004based therapy

Steroid + IVIG

Steroid

Only observation

Hematopoietic stem cell transplantation

Causes of $\mathrm{HLH}$

EBV-associated

Immunodeficiency

Autoimmune disease (AID)

Unknown cause and infection other than EBV

Treatment response at 8 weeks

Early stable responder

Late stable responder

Unstable responder

Non-responder

Abbreviations: HLH, hemophagocytic lymphohistiocytosis; AST, aspartate aminotransferase; ALT, alanine transferase; LDH, lactate dehydrogenase; CRP, C-reactive protein; DIC, disseminated intravascular coagulation; EBV, Epstein-Barr virus; CR, complete remission; EBV, Epstein-Barr virus; TG, triglyceride; GGT, gamma-glutamyl transpeptidase.

\section{Treatment outcomes}

A total of 83/88 (94.3\%) patients were treated. 65/88 (73.8\%) (unknown cause $n=18$, EBV infection $n=$ 20, infection other than EBV $n=18$, autoimmune disease $n=3$, immunodeficiency $n=6$ ) patients were administered therapy according to the HLH94 protocol. 5/88 (5.6\%) patients were administered dexamethasone plus gamma globulin. 12/88 (13.6\%) patients were administered dexamethasone alone. 1/88 (1.1\%) patient underwent HCT. Dexamethasone alone with no further treatment was administered to patients who had mild symptoms and showed rapid improvement. 5/88 (5.6\%) patients were not treated. 
Of these, 3 patients were without signs of HLH activity, and 2 patients died. Cyclosporine and etoposide were not administered to patients with substantially altered organ function or who were in a generally poor condition. 57/88 (64.7\%) patients achieved complete response; of these, 30 patients achieved complete response at 4 weeks after treatment and 27 achieved complete response at 8 weeks or later. 3 patients relapsed; of these, 1 patient relapsed 8 weeks after treatment, 1 patient relapsed 4 months after treatment, and 1 patient relapsed 12 months after treatment. Relapsed patients had EBV-HLH, and were treated with rituximab $(n=1)$, alemtuzumab $(n=1)$, or allogeneic-HCT $(n=1)$ according to the HLH-94 protocol. Regarding dynamic response, $32 / 88$ (36.4\%) patients were early stable responders, $28 / 88$ (31.8\%) were late stable responders, $14 / 88(15.9 \%)$ were unstable responders, and 13/88 (14.7\%) were primary refractory non-responders.

\section{Survival outcomes according to treatment response}

5-year OS rates for patients with EBV-HLH and HLH due to an unknown cause and infections other than EBV were $80.0 \%$ and $77 \%$, respectively, and higher than in patients with $\mathrm{HLH}$ from other causes (Fig. $1 \mathrm{~A}$ ).

Among all patients, 5-year OS rates for patients who achieved complete response at 4 weeks $(n=30)$ and 8 weeks $(n=57)$ were each 100\%.5-year OS rates for patients who achieved partial response at 4 weeks $(n=38)$ and 8 weeks $(n=14)$ were $86 \%$ and $44.0 \%$, respectively (Fig. 1B-C). 5-year OS rates for early stable responders and late stable responders were each 100\% (Fig. 1D).

Among patients with EBV-HLH, 5-year OS rates for patients who achieved an early stable response, late response or non response were $100 \%, 100 \%$, and $0.0 \%$, respectively.

Prognostic factors and risk stratification

On univariate survival analysis, hemoglobin level, PLT, and albumin level at diagnosis, and dynamic treatment response were significantly correlated with survival outcomes. On multivariate analysis, nonresponse at 8 weeks was the most powerful predictor of poor OS. When treatment response was excluded, hemoglobin $<60 \mathrm{~g} / \mathrm{L}$ and albumin $<25 \mathrm{~g} / \mathrm{L}$ at diagnosis were associated with poor OS (Table 2). In patients with EBV-HLH, hemoglobin $<60 \mathrm{~g} / \mathrm{L}$ at diagnosis was associated with poor OS. 
Table 2

Univariate and multivariate analysis of parameters affecting OS in non-malignancy associated HLH

\begin{tabular}{|c|c|c|c|c|c|c|}
\hline & Univariate & lysis & Multivariate anal & & & \\
\hline & & & $\begin{array}{l}\text { Treatment respon } \\
\text { included }\end{array}$ & & $\begin{array}{l}\text { Treatment respon } \\
\text { excluded }\end{array}$ & \\
\hline 5-year OS & P & $\mathrm{HR}(95 \% \mathrm{Cl})$ & $\mathrm{PHR}(95 \% \mathrm{Cl})$ & $\mathrm{P}$ & & \\
\hline Age $<1$ years old & $\begin{array}{l}80.0 \% \text { VS } \\
(69.0 \%)\end{array}$ & 0.425 & & & & \\
\hline EBV-associated & $\begin{array}{l}79.0 \% \text { VS } \\
(67.0 \%)\end{array}$ & 0.262 & & & & \\
\hline Hemoglobin $<60 \mathrm{~g} / \mathrm{L}$ & $\begin{array}{l}25.0 \% \text { VS } \\
(77.0 \%)\end{array}$ & $<0.001$ & & & $\begin{array}{l}3.22(2.01- \\
19.28)\end{array}$ & 0.002 \\
\hline Platelet $<30 \times 10^{\wedge} 9 / \mathrm{L}$ & $\begin{array}{l}36.0 \% \text { VS } \\
(79.0 \%)\end{array}$ & $<0.001$ & & & $2.15(0.88-5.24)$ & 0.093 \\
\hline ALT $>200 \mathrm{U} / \mathrm{L}$ & $\begin{array}{l}73.0 \% \text { VS } \\
(71.0 \%)\end{array}$ & 0.935 & & & & \\
\hline$T \mathrm{TG}>2.5 \mathrm{mg} / \mathrm{dl}$ & $\begin{array}{l}76.0 \% \text { VS } \\
(62.0 \%)\end{array}$ & 0.173 & & & & \\
\hline Fibrinogen $<1.5$ & $\begin{array}{l}66.0 \% \text { VS } \\
(80.0 \%)\end{array}$ & 0.170 & & & & \\
\hline Ferritin $>10000$ & $\begin{array}{l}75.0 \% \text { VS } \\
(72.0 \%)\end{array}$ & 0.744 & & & & \\
\hline Albumin $<25 \mathrm{~g} / \mathrm{L}$ & $\begin{array}{l}33.0 \% \text { VS } \\
(82.0 \%)\end{array}$ & $<0.001$ & & & $7.71(3.10-19.19)$ & $<0.001$ \\
\hline $\begin{array}{l}\text { Dynamic treatment } \\
\text { response }\end{array}$ & & 0.010 & & 0.010 & & \\
\hline Early stable responder & $100 \%$ & & 0.00 & & & \\
\hline Late stable responder & $100 \%$ & & 0.00 & & & \\
\hline Unstable responder & $36 \%$ & & $0.21(0.08-0.52)$ & & & \\
\hline Non-responder & $0 \%$ & & 1.0 & & & \\
\hline
\end{tabular}

A prognostic risk score was established and weighted based on hazard ratios calculated for three parameters measured at diagnosis: hemoglobin $<60 \mathrm{~g} / \mathrm{L}$ ( 2 points), platelets $<30 \times 10^{9} / \mathrm{L}$ (1 point), albumin $<25 \mathrm{~g} / \mathrm{L}$ (2 points). Five-year OS of low-risk (score $0-1$ ), intermediate-risk (score 2), and poor-risk (score $\geq 3$ ) patients were $88 \%, 38 \%$, and $22 \%$, respectively (Fig. 2 ).

\section{Discussion}

This study analyzed treatment response and prognostic factors in pediatric patients with secondary HLH after excluding MA-HLH. To the authors' knowledge, this is the largest existing dataset reporting on prognostic factors in children with secondary HLH without malignancies. Findings showed that EBV was the most common cause of pediatric secondary HLH not associated with malignancy. Patients with EBVHLH showed a better treatment response and improved OS compared to children with secondary HLH due to other causes. In contrast to these data, a previous study in adult patients with HLH reported that EBV was associated with poor survival outcomes. The overall response to treatment at 4 weeks was similar in patients with HLH caused by EBV, autoimmune disease, other infections, or unknown causes, but decreased in the EBV group at 8 weeks[15]. The disparate results between this previous report in adults and the present study may have been caused by misdiagnosis of EBV in our pediatric population. 
Notably, in the present study, pediatric patients with HLH caused by chronic active EBV infection had a very poor prognosis, which is consistent with the previous report in adults[15].

Urgent preparation for allogeneic HCT should be considered for high-risk patients at HLH diagnosis. In the present study, prognosis was very poor in patients with HLH caused by autoimmune diseases and immunodeficiency. HLH that results from rheumatic or other systemic diseases, including Still's disease and sarcoidosis, is termed macrophage activation syndrome (MAS)[16-18]. In our cohort, 13 patients had HLH caused by autoimmune disease, including systemic lupus erythematosus, juvenile rheumatoid arthritis, dermatomyositis, Kawasaki disease, and anaphylactoid purpura. There were 6 patients with HLH caused by immunodeficiency, including chronic granuloma, X-linked lymphocyte proliferative diseases, and humoral immune deficiency. A previous report found that most patients with primary immunodeficiencies other than cytotoxicity defects or X-linked lymphoproliferative disorders presenting with conditions fulfilling current criteria for $\mathrm{HLH}$ had chronic granulomatous disease with hemophagocytic episodes mainly associated with bacterial infections. HLH was not found in other congenital defects of phagocytes, and was rarely found in other congenital immune defects. The authors proposed that HLH syndrome in chronic granulomatous disease not only reflects an impaired response to infection, but also a genetic predisposition to the inflammatory reaction[19]. 5-year OS rates for early stable responders and late stable responders were each $100 \%$, indicating that patients can experience long-term survival if they fail to achieve a complete response at 4 weeks after treatment but exhibit a continuous and stable response until 8 weeks. The 5-year OS rate for patients who achieved partial response at 8 weeks was low at $44.0 \%$. On multivariate analysis, non- response at 8 weeks was the most powerful predictor of poor OS. When treatment response was excluded, hemoglobin $<60 \mathrm{~g} / \mathrm{L}$ and albumin $<25 \mathrm{~g} / \mathrm{L}$ at diagnosis were associated with poor OS. In previous reports in adult patients with $\mathrm{HLH}$, one study associated hypofibrinogenemia ( $\leq 150 \mathrm{mg} / \mathrm{dl}$ ), fibrinogen $\leq 200 \mathrm{mg} / \mathrm{dl}(P=0.04)$, and prothrombin time $>50 \%$ with increased mortality[20], while another showed that patients with disseminated intravascular coagulation, nosocomial infections and neurological symptoms had a statistically significant worse survival[20].

$\mathrm{HLH}$ is characterized by a hyperinflammatory phenotype[21-22]. As clinical criteria for HLH include nonspecific findings that overlap with other diseases, HLH may be an obsolete term that is better replace by hyperinflammatory syndrome and/or hypercytokinemia[22-23]. The $\mathrm{HLH}-94$ treatment protocol includes 8 weeks of initial therapy that aims to achieve clinical remission, followed by continuation therapy that aims to keep patients alive and stable until an acceptable bone marrow transplantation donor becomes available[4]. In the present study, 22 (25\%) patients received dexamethasone alone and $23(26.1 \%)$ patients did not receive any treatment; these patients had a survival rate $>90 \% .13$ patients received continuation therapy; among these, 7 patients achieved complete response, 5 patients achieved partly response and 1 patient was treated with allogeneic HCT. These findings show that pediatric patients with stable disease can be treated with glucocorticoids alone or watchful waiting, but children with high-risk factors or disease progression require treatment according to the HLH94 protocol. 
$\mathrm{HLH}$ is a rare condition, and rigorously conducted prospective studies are lacking.There remains an unmet need to identify prognostic factors for secondary HLH, as few data are available for riskstratification. The present study aimed to fill this evidence gap, but it was associated with several limitations. First, it was a retrospective study; despite this, medical records showed that patients were consistently treated and managed, and the sample size was adequate. Second, the risk-stratification model should be validated by larger studies. Third, there was significant heterogeneity in this cohort, in terms of attributed causes but also disease severity, since a number of patients were not treated. However, exclusion of cases of MA-HLH ensured that treatment response to the protocols and survival outcomes were applicable to risk- stratification.

\section{Conclusions}

This is the first study to identify prognostic factors in children with non- malignancy associated secondary HLH. Findings suggest that clinicians should be aware of predictive factors at diagnosis, including hemoglobin $<60 \mathrm{~g} / \mathrm{L}$ and albumin $<25 \mathrm{~g} / \mathrm{L}$, and consider treatment response at 8-weeks as a criterion to identify patients at high-risk of disease progression and the need for second-line therapy and allogeneic HCT.

\section{Abbreviations}

HLH:Hemophagocytic lymphohistiocytosis; ALL:Acute lymphoblastic leukemia; AML:Acute myeloblastic leukemia; IVIG:Intravenous Immunoglobulin; Allo HSCT:Allogeneic Hematopoietic stem cell transplantation; OS:Overall survival; MA-HLH:malignancy-associated HLH; CBC:complete blood cell; PT:prothrombin time; AST:aspartate aminotransferase; ALT:alanine aminotransferase; LDH:lactate dehydrogenase;MAS:macrophage activation syndrome .

\section{Declarations}

\section{Acknowledgements}

We thank Professor Yili WU for providing the medical statistics of the data.

\section{Funding}

This study was funded by Li-rong Sun.

\section{Availability of data and materials}

Data are available from the authors upon reasonable request.

\section{Authors'contributions}


HP conceptualized and designed the study, and reviewed and revised the manuscript. GYWand EBG drafted the initial manuscript. LS and AQS collected data. XDL, ZY and LRS was responsible for the follow-up. All authors approved the final manuscript as submitted and agree to be accountable for all aspects of the work.

\section{Competing interests}

The authors declare that they have no competing interests.

\section{Consent for publication}

Not applicable.

\section{Ethics approval and consent to participate}

A copy of the written consent form is available for review by the editor of this journal. The study has been performed with the approval of the Ethics Committee of the Affiliated University of Qingdao University(no: 20190113). The protocol has been designed and the study will be conducted in line with the principles of the Declaration of Helsinki (1964).

\section{Authors' Affiliations}

(1) Department of Paediatric Hematology, Affiliated Hospital of Qingdao University, Qingdao, China.

(2) Correspondence to: Li-rong Sun, Department of Pediatric Hematology, Affiliated Hospital of Qingdao University, 16 Jiangsu Road, Qingdao, Shandong 266003, China. E-mail: sunlr@vip.sina.com.

\section{References}

1. Janka GE, Lehmberg K. Hemophagocytic syndromes. Blood Rev. 2014; 28(4):135-42.

2. Janka GE. Familial and Acquired Hemophagocytic Lymphohistiocytosis. Annu Rev Med.2012; 63:233- 246.

3. Jordan MB,Allen CE,Greenberg J,et al.Challenges in the diagnosis of hemophagocytic lymphohistiocytosis: Recommendations from the North American Consortium for Histiocytosis (NACHO).Pediatr Blood Cancer.2019;66(11):e27929.

4. Kleynberg RL, Schiller GJ.Secondary hemophagocytic lymphohistiocytosis in adults: an update on diagnosis and therapy.Clin Adv Hematol Oncol.2012;10(11):726-732.

5. Apodaca E, Rodríguez-Rodríguez S,Tuna-Aguilar EJ, et al.Prognostic Factors and Outcomes in Adults With Secondary Hemophagocytic Lymphohistiocytosis: A Single-center Experience. Clin Lymphoma Myeloma Leuk.2018;18(10):e373-e380.

6. Ho Yoon J, Soo Park S, Woo Jeon Y, et al.Treatment outcomes and prognostic factors of adult patients with non-malignancy associated secondary hemophagocytic lymphohistiocytosis. 
Haematologica.2019; 14(2):269-276.

7. Takahashi N, Chubachi A, Kume M, et al. A clinical analysis of 52 adult patients with hemophagocytic syndrome: the prognostic significance of the underlying diseases. Int J Hematol.2001;74(2):209-213.

8. Li J, Wang Q, Zheng W, et al. Hemophagocytic lymphohistiocytosis: clinical analysis of 103 adult patients. Medicine.2014;93(2):100-105

9. Marsh RA, Jordan MB, Talano JA, et al. Histiocyte Society Salvage Therapy Working Group. Salvage therapy for refractory hemophagocytic lymphohistiocytosis: a review of the published experience. Pediatr Blood Cancer.2017;64(4).

10. Ehl S, Astigarraga I, von Bahr Greenwood T,et al. Recommendations for the use of etoposide-based therapy and bone marrow transplantation for the treatment of $\mathrm{HLH}$ : consensus statements by the HLH Steering Committee of the Histiocyte Society.J Allergy Clin Immunol Pract.2018;6(5):1508-1517.

11. Henter JI, Samuelsson-Horne A, Arico M, et al. Treatment of hemophagocytic lymphohistiocytosis with HLH-94 immunochemotherapy and bone marrow transplantation. Blood.2002;100(7):23672373.

12. Cooper N, Rao K, Gilmour K, et al. Stem cell transplantation with reduced-intensity conditioning for hemophagocytic lymphohistiocytosis. Blood.2006;107(3):1233-1236.

13. Machaczka M, Nahi H, Karbach $H$, et al. Successful treatment of recurrent malignancyassociated hemophagocytic lymphohistiocytosis with a modified HLH-94 immunochemotherapy and allogeneic stem cell transplantation. Med Oncol.2012;29(2):1231-1236.

14. 14. Rosée PL, Horne A, Hines M, et al. Recommendations for the management of hemophagocytic lymphohistiocytosis in adults.Blood.2019;133(23):2465-2477.

15. Yoon JH, Park SS, Jeon YW囚et al. Treatment outcomes and prognostic factors in adult patients with secondary hemophagocytic lymphohistiocytosis not associated with malignancy. Haematologica.2019;104(2):269-276.

16. Hadchouel M, Prieur AM, Griscelli C. Acute hemorrhagic, hepatic,andneurologic manifestations in juvenile rheumatoid arthritis: possible relationship to drugs or infection. JPediatr.1985;106(4):561566.

17. 17. Ravelli A, Davi S, Minoia F,et al. Macrophage activation syndrome. HematolOncolClinNorth Am.2015;29(5):927-941.

18. Abughanimeh O, Qasrawi A, Abu Ghanimeh M. Hemophagocytic Lymphohistiocytosis Complicating Systemic Sarcoidosis. Cureus. 2018;10(6):e2838.

19. 19. Bode SF, Ammann S, Al-Herz W, et al.The syndrome of hemophagocytic lymphohistiocytosis in primary immunodeficiencies:implications for differential diagnosis and pathogenesis. Haematologica.2015;100(7):978-988.

20. Valade S, Azoulay E, Galicier L.Coagulation Disorders and Bleedings in Critically III Patients With Hemophagocytic Lymphohistiocytosis. Medicine (Baltimore).2015;94(40):e1692. 
21. Otrock ZK1, Eby CS.Clinical characteristics, prognostic factors, and outcomes of adult patients with hemophagocytic lymphohistiocytosis. Am J Hematol.2015;90(3):220-224.

22. Teachey DT, Rheingold SR, Maude SL, et al.Cytokine release syndrome after blinatumomab treatment related to abnormal macrophage activation and ameliorated with cytokine-directed therapy.Blood.2013;121(26):5154-5157.

23. Otrock ZK, Daver N, Kantarjian HM, et al.Diagnostic Challenges of Hemophagocytic Lymphohistiocytosis. Clin Lymphoma Myeloma Leuk.2017;17S:S105-S110.

\section{Figures}



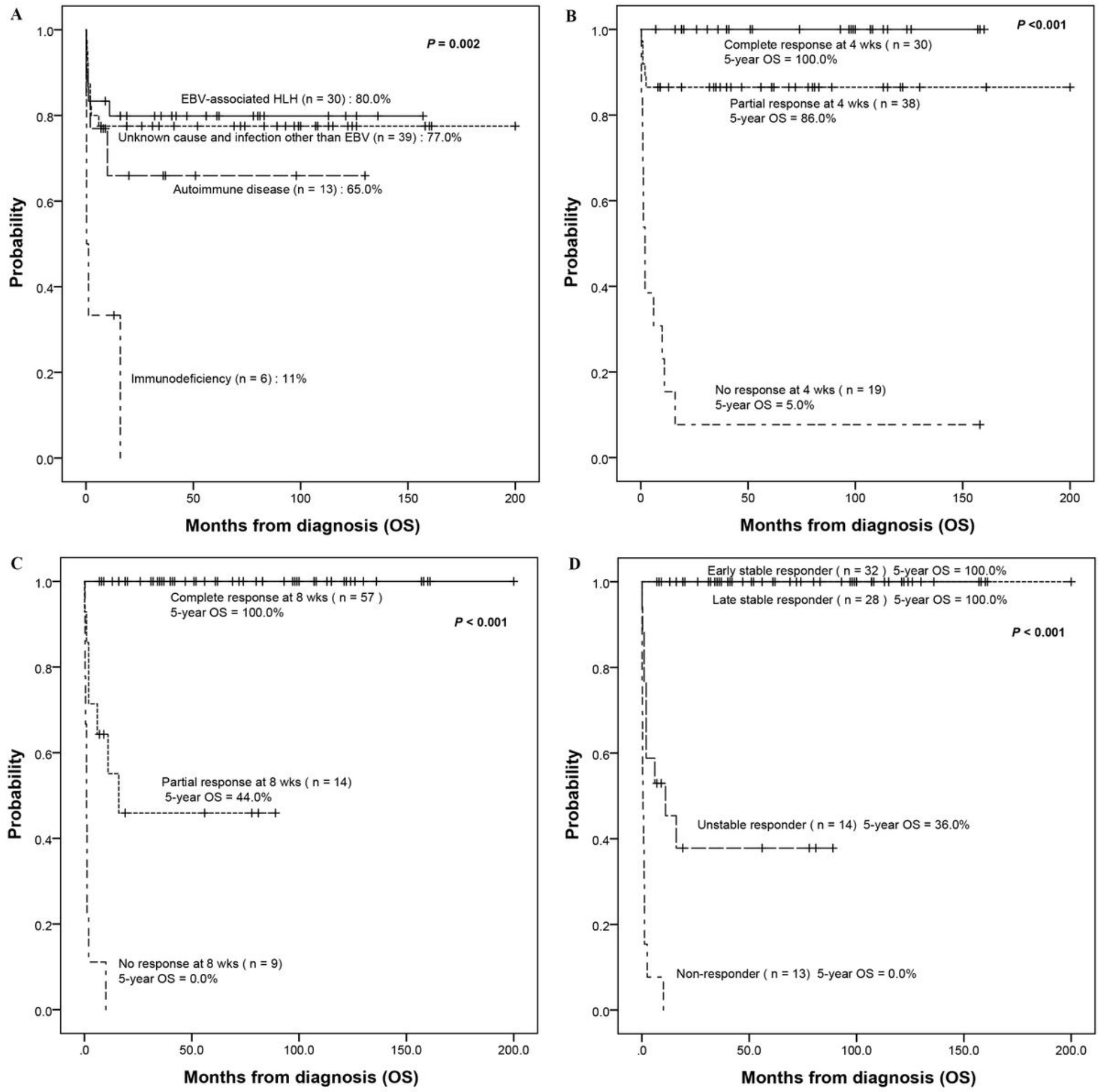

\section{Figure 1}

Survival outcomes according to the cause of secondary HLH and treatment response. A. The cause of secondary HLH. B.Treatment response at 4 weeks. C. Treatment response at 8 weeks. D. Dynamic treatment responses at 8 weeks. 


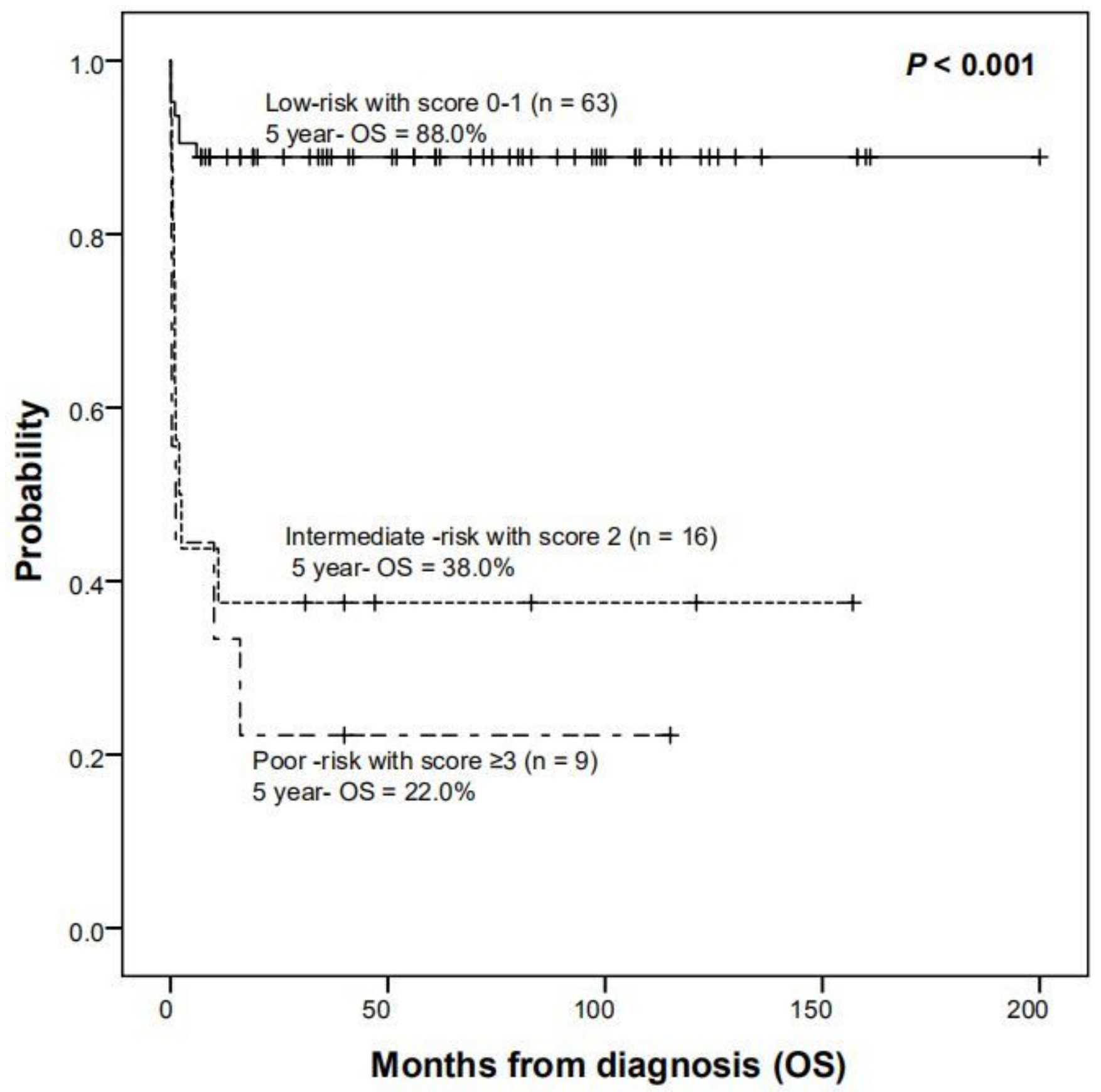

Figure 2

Survival outcomes according to HLH risk-score (low-risk [score 0-1], intermediate-risk [score 2], and highrisk [score $\geq 3]$ ). 